\title{
Um Retrato da Área de Neurociência e Comportamento no Brasil
}

\author{
Dora Fix Ventura ${ }^{1}$ \\ Universidade de São Paulo
}

\begin{abstract}
RESUMO - A neurociência compreende o estudo do controle neural das funções vegetativas, sensoriais e motoras; dos comportamentos de locomoção, reprodução e alimentação; e dos mecanismos da atenção, memória, aprendizagem, emoção, linguagem e comunicação. Tem, portanto, uma importante área de interface com a Psicologia. Dentre seus objetivos, a neurociência busca esclarecer os mecanismos das doenças neurológicas e mentais por meio do estudo do sistema nervoso normal e patológico. Sua evolução no Brasil tem ocorrido desde meados do século passado, e seu desenvolvimento foi incentivado pela criação de sociedades científicas específicas. O presente artigo relata esse desenvolvimento e descreve os principais grupos atuantes na neurociência brasileira.
\end{abstract}

Palavras-chave: neurociência; Psicologia; sistema nervoso; comportamento; doença mental; sociedades científicas.

\section{A Report on the Area of Neuroscience and Behavior in Brazil}

\begin{abstract}
Neuroscience comprises the study of the neural control of vegetative, sensory and motor functions; of the locomotion, reproduction and feeding behaviors; and of the mechanisms of attention, memory, learning, emotion, language and communication. It has, therefore, an important area of interface with Psychology. Among its goals, neuroscience seeks to explain the mechanisms of neurologic and mental diseases through the study of the normal and pathologic nervous system. Its evolution in Brazil has occurred since the middle of the past century, and its development was encouraged by the creation of specific scientific societies. The present article describes this development and the main active groups in Brazilian neuroscience.
\end{abstract}

Keywords: neurosciences; Psychology; nervous system; behavior; mental disease; scientific societies.

A neurociência compreende o estudo do sistema nervoso e suas ligações com toda a fisiologia do organismo, incluindo a relação entre cérebro e comportamento. $O$ controle neural das funções vegetativas - digestão, circulação, respiração, homeostase, temperatura -, das funções sensoriais e motoras, da locomoção, reprodução, alimentação e ingestão de água, os mecanismos da atenção e memória, aprendizagem, emoção, linguagem e comunicação, são temas de estudo da neurociência.

\section{Relevância da Área}

Modelos computacionais que simulam as funções acima mencionadas, notadamente as funções locomotoras e sensoriais, a aprendizagem e a memória, são objeto de pesquisa em inteligência artificial e visão artificial. Ferramentas da informática são usadas para entender os sistemas biológicos e soluções inspiradas na biologia e na psicologia são desenvolvidas para aplicações que servem a diferentes ramos da engenharia, tais como sensoriamento remoto, controle de sistemas de produção industrial, linha de montagem de fábricas e muitas outras aplicações.

1 Endereço para correspondência: Instituto de Psicologia, Universidade de São Paulo. Av. Prof. Mello Moraes, 1721 Bloco A, sala D9, Cidade Universitária. São Paulo, SP. CEP 05508-030.E-mail: dventura@usp.br.
A neurociência está em grande expansão, e foi eleita com destaque pelo Governo dos EUA como prioritária na década de 1990, que ficou conhecida como a 'Década do Cérebro' (Library of Congress, 2010). Muitos também consideram o século 21 o século do cérebro, no qual as grandes conquistas da humanidade estarão dirigidas para a compreensão das funções neurais humanas.

A neurociência estuda também as doenças do sistema nervoso e seus reflexos em todas as funções do indivíduo, procurando métodos de diagnóstico, prevenção e tratamento, além da descoberta das causas e mecanismos. Desde uma dor de cabeça até a doença de Alzheimer, o custo social e econômico dessas afecções é imenso, e é também muito grande a parcela da economia dedicada ao desenvolvimento e à produção e comercialização de fármacos e de equipamentos de diagnóstico. $\mathrm{O}$ aprofundamento da pesquisa traz o reconhecimento de novas doenças, permitindo seu estudo e tratamento. São muitos os exemplos de novas descobertas. No século XIX, Freud descreveu a paralisia cerebral, tendo usado esse nome para distinguí-la da paralisia infantil. Nas últimas décadas foi reconhecida a síndrome do pânico e há menos de 10 anos, a morte súbita, as graves consequências de distúrbios do sono e os príons e seu possível envolvimento em patologias neurológicas e mentais. É surpreendente pensar que as doenças do sono, que sempre afligiram a humanidade, só tiveram reconhecimento muito recentemente. 


\section{Doenças Neurológicas e Mentais: Aspectos Epidemiológicos e Impacto Econômico}

A pesquisa em neurociência visa, dentre seus objetivos, esclarecer os mecanismos das doenças neurológicas e mentais por meio do estudo do sistema nervoso normal e patológico.

As funções do sistema nervoso podem ser alteradas por eventos ambientais como trauma, agentes infecciosos ou tóxicos; por tumores, mutações gênicas e defeitos congênitos; por eventos vasculares e deficiências nutricionais, e por muitos outros fatores. Muitas doenças do sistema nervoso são totalmente incapacitantes, outras provocam prejuízos de diferentes níveis de gravidade. Infelizmente, não temos acesso a dados de prevalência brasileiros. Para exemplificar alguns números mais relevantes são citadas a seguir informações dos EUA.

Dados do National Institute of Health - NIH (2002) mostram que, na população dos EUA, dentre as doenças neurológicas que em geral se instalam na infância: (1) a epilepsia afeta cerca de 2,5 milhões de norte-americanos; (2) a paralisia cerebral ocorre em 750.000 nascimentos; (3) o autismo atinge 400.000 crianças; e (4) os tumores cerebrais são a segunda causa de morte por câncer em crianças até 15 anos. Dentre as doenças que afetam adultos, causando anos de invalidez, perda de vida e impacto econômico, os dados do NIH indicam que: (1) acima dos 60 anos, a demência do tipo Alzheimer, o acidente vascular encefálico e a doença de Parkinson são frequentes; (2) os acidentes vasculares encefálicos (cerebrais) são a terceira entre todas as causas de morte e uma causa importante de invalidez (mais de 700.000 americanos são afetados); (3) o trauma cerebral e as lesões medulares ocorrem principalmente antes dos 30 anos, sendo o trauma cerebral responsável por mais de 5,3 milhões de comprometimentos e mais de 50.000 óbitos; (4) a esclerose múltipla começa por volta dos 30 anos de idade. Finalmente, a dor é a principal queixa para a procura de atendimento médico em geral. A prevalência de dor crônica é alta e suas causas difíceis de tratar.

A neurociência contribui também para o esclarecimento das doenças mentais. O National Institute of Mental Health - NIMH (2002) define doenças mentais como aquelas em que há alteração no pensamento, humor ou comportamento (isoladamente ou combinados), acarretando sofrimento e/ou prejuízo funcional. Dados daquela agência mostram que durante um período de um ano, $22 \%$ da população americana adulta (44 milhões de pessoas) têm alguma doença mental diagnosticável por critérios confiáveis; dentre essas pessoas, $3 \%$ associam o abuso de drogas à doença mental.

O prejuízo causado por uma patologia pode ser calculado de várias formas. O índice AVAI (Anos de Vida Ajustados por Incapacidade, do inglês Disability Adjusted Life Years - DALY) computa os anos de vida com saúde perdidos por causa da doença, seja por morte prematura ou incapacitação, e leva em conta a gravidade da incapacitação. Por exemplo, depressão profunda compara-se, no AVAI, à cegueira ou paraplegia, enquanto a psicose ativa na esquizofrenia equivale à tetraplegia. Dados da Organização Mundial da Saúde - OMS (do inglês World Health Organization - WHO; 2002) mostraram que dentre as patologias de maior prevalência, os prejuízos acarretados por doenças mentais são superiores aos das doenças cardiovasculares (ver Tabela 1). Além do sofrimento e incapacidade do indivíduo, essas doenças também representam custos diretos em recursos médicos e custos indiretos em perda de produtividade.

As doenças do sistema nervoso são muito difíceis de serem tratadas, mas têm havido progressos notáveis nos últimos anos, decorrentes do grande esforço de pesquisa em neurociência. Os progressos mais significativos são destacados pelo NIH (2002), em sua justificativa orçamentária: melhores técnicas diagnósticas a partir da genética molecular e neuroimagem; tratamentos para acidente vascular encefálico e para lesões da medula espinhal; novas drogas para esclerose múltipla, epilepsia e doença de Parkinson; aparelhos eletromecânicos para deficiências sensoriais e motoras; prevenção de acidente vascular encefálico e defeitos neurológicos congênitos. São, ainda, listados pelo documento do NIH tratamentos promissores para doenças do sistema nervoso em estudo, incluindo drogas, vacinas, estimulação elétrica, transplantes celulares, fatores de crescimento naturais, próteses neurais, terapia gênica e intervenções comportamentais. Verifica-se, com esses progressos, que a área de neurociência é importante para a Psicologia, tanto na pesquisa básica como na atividade clínica.

Tabela 1. Prejuízo em categorias selecionadas de patologias.

\begin{tabular}{lc}
\hline Categorias de Patologias & $\begin{array}{c}\text { AVAI } \\
\text { Total (\%) }\end{array}$ \\
\hline Todas as doenças infecciosas e parasitárias & 24 \\
Todas as doenças mentais & 13 \\
Todas as doenças cardiovasculares & 10 \\
Todo o uso de álcool e drogas & 7 \\
Todas as doenças respiratórias & 6 \\
Todas as doenças malignas (câncer) & 5 \\
\hline
\end{tabular}

Fonte: WHO, 2002

\section{Sociedades Científicas no Brasil}

A neurociência no Brasil está representada principalmente pela Sociedade Brasileira de Neurociências e Comportamento ( $\mathrm{SBNeC}$ ), que congrega a pesquisa básica da área. A produção neurocientífica está também presente nas Sociedades Brasileiras de Psicologia, de Farmacologia, de Fisiologia, de Bioquímica, e na Brazilian Research Association on Vision and Ophthalmology. Na área clínica, a neurociência brasileira é apresentada nas Sociedades Brasileiras de Neurologia, de Psiquiatria e de Neuropsicologia.

A $\mathrm{SBNeC}$, originalmente Sociedade Brasileira de Psicobiologia, tem 34 anos de existência. Foi fundada por Elisaldo Carlini, que reuniu um grupo de psicólogos, 
psicofarmacólogos, neurofisiologistas, psiquiatras e outros especialistas. A ata de fundação gerada nessa reunião histórica mostra a argumentação desses pesquisadores na época, muito interessados em promover a integração entre psicologia e neurociência. Esse grupo decidiu pela fundação da nova sociedade a partir da Sociedade Latinoamericana de Psicobiologia, cujo sucesso tinha sido comprometido pelas dificuldades políticas dos países da região na década de 1970. O nome Sociedade Brasileira de Psicobiologia perdurou até os anos 1990, quando, para evitar a criação de uma segunda sociedade na mesma área, dedicada à neuroquímica, decidiu-se mudar o nome da entidade para Sociedade Brasileira de Neurociências e Comportamento. A manutenção da palavra comportamento como parte do nome tem grande relevância para a área de Psicologia, pois constitui a forma de integrar os psicólogos experimentais na sociedade, valorizando o enfoque no estudo do comportamento. Essa ênfase espelha a motivação original do grupo fundador de integrar psicólogos, estudiosos do comportamento, com os demais ramos da fisiologia, farmacologia, morfologia, e outras áreas de estudo do sistema nervoso.

\section{A Neurociência no Brasil}

A pesquisa em neurociência tem sólida tradição e ampla representação em nosso país (Silveira, 2004; Timo-Iaria, s.d.; Ventura, 1997). A partir das décadas de 40 e 50 do século passado, a área recebeu grande impulso com Aristides Pacheco Leão e Hiss Martins Ferreira estudando o fenômeno de depressão cortical alastarante na Universidade Federal do Rio de Janeiro (UFRJ); Carlos Ribeiro Diniz isolando e caracterizando o veneno de escorpião na Universidade Federal de Minas Gerais (UFMG); e Miguel Covian estabelecendo um grupo de pesquisa em eletrofisiologia do sistema nervoso na Faculdade de Medicina da Universidade de São Paulo em Ribeirão Preto (USP-RP).

Na década de 1970, outros grupos iniciaram novas direções de pesquisa em neurociência: Carlos Eduardo Rocha Miranda e Eduardo Oswaldo Cruz fundaram seus laboratórios para o estudo do sistema visual na UFRJ, Cesar Timo-Iaria iniciou trabalhos no controle neural do metabolismo e em mecanismos de atenção e sono, Elisaldo Araújo Carlini criou um grupo de psicofarmacologia na Escola Paulista de Medicina e psicólogos experimentais e etólogos, em torno de Carolina Bori e Walter H. A. Cunha, começaram a trabalhar na USP e na Universidade de Brasília (UnB). Essas origens se refletem nos grupos de neurociência que existem hoje.

Os temas de pesquisa dos principais grupos de neurociência no Brasil são apresentados a seguir (e resumidos na Tabela 2):

- Memória em humanos e não humanos, investigada por pesquisadores do Centro de Memória do Departamento de Bioquímica da UFRGS, do Instituto de Biociências da USP, dos departamentos de Psicobiologia e de Fisiologia da Universidade Federal de São Paulo (UNIFESP) e do Departamento de Psicologia da USPRP. Esses grupos integram neuroquímica, fisiologia, farmacologia, neuroimagem e comportamento para estudar como humanos e não humanos adquirem, armazenam e recuperam informações.

- Ansiedade e depressão, estudadas principalmente pelo grupo do Departamento de Psicobiologia da USPRP, focalizando o papel da serotonina na modulação das respostas de medo e ansiedade em humanos e não humanos. Os trabalhos básicos do grupo constituíram referência para toda uma geração de drogas serotonérgicas. Com o mesmo tipo de metodologia, outros aspectos de ansiedade e estados emocionais, como a depressão, têm sido estudados pelos grupos do Departamento de Psicobiologia da UNIFESP, da Faculdade de Medicina da Universidade de Campinas (UNICAMP) e do Departamento de Psicologia Experimental do Instituto de Psicologia da USP. Outros aspectos da psicofarmacologia são estudados por grupos das universidades federais do Espírito Santo (UFES), Paraná (UFPR), Rio Grande do Norte (UFRN) e Santa Catarina (UFSC).

- Etologia, pesquisada pelo Departamento de Psicologia Experimental do Instituto de Psicologia da USP, pelo Instituto de Biociências da UNICAMP, pelo Departamento de Zoologia da UFMG e pelo Departamento de Psicobiologia da UFRN.

- Epilepsia, investigada no Departamento de Neurologia e Neurocirurgia da UNIFESP, o que resultou na criação de um novo modelo animal de crises epilépticas espontâneas recorrentes, agora amplamente utilizado em todo o mundo. Posteriormente, passou a ser estudada também no Departamento de Fisiologia da UNIFESP, nos departamentos de Fisiologia e Neurologia da Faculdade de Medicina da USP-RP e nos departamentos de Fisiologia da UFPR e da UFMG. Epilepsia é também estudada por meio de análise neuroetológica no Departamento de Psicobiologia da USP-RP.

- Sistema visual, incluindo aspectos morfológicos, neuroquímicos, eletrofisiológicos, de psicofísica (humana e não humana) e genéticos, estudado por um dos mais numerosos conjuntos de pesquisadores. Os estudos de desenvolvimento do sistema visual no gambá e de eletrofisiologia e morfologia da visão em primatas, realizados no Departamento de Neurobiologia do Instituto de Biofísica Carlos Chagas Filho da UFRJ, deram origem a outros grupos no Centro de Ciências Biológicas da UnB, no Núcleo de Medicina Tropical e no Centro de Ciências Biológicas da Universidade Federal do Pará (UFPA), e no Departamento de Neurobiologia da Universidade Federal Fluminense (UFF). Outros aspectos do funcionamento do sistema visual, estudados em abelhas, peixe, tartaruga, roedores e primatas humanos e não humanos, foram abordados por meio de psicofísica e eletrofisiologia, e metodologias morfológicas, moleculares e celulares, da retina e vias visuais. Esses aspectos têm sido investigados por grupos do Departamento de Psicologia Experimental do Instituto de Psicologia da USP, dos Departamentos de Fisiologia e Biofísica e de Biologia Celular e Tecidual do Instituto de Ciências Biomédicas da USP, no Departamento de Psicobiologia da Faculdade de Filosofia Ciências e Letras da USP-RP, no Departamento 
Tabela 2. Principais temas e grupos de pesquisa em neurociência no Brasil.

\begin{tabular}{|c|c|c|}
\hline Tema & Departamento & Universidade \\
\hline \multirow{3}{*}{ Memória } & Bioquímica & ICB-UFRGS \\
\hline & Fisiologia & IB-USP \\
\hline & Psic. Experimental & IP-USP \\
\hline \multirow{5}{*}{$\begin{array}{l}\text { Psicofarmacologia (ansiedade, depressão, modelos animais } \\
\text { para doença mental, propriedades farmacológicas de produ- } \\
\text { tos naturais) }\end{array}$} & Psicobiologia & UNIFESP \\
\hline & Psicobiologia & FFCLRP-USP \\
\hline & Farmacologia & UFSC \\
\hline & Psic. Experimental & IP-USP \\
\hline & Fisiologia & FM-UNICAMP \\
\hline \multirow{8}{*}{$\begin{array}{l}\text { Sistema visual (morfologia, neuroquímica, eletrofisiologia, } \\
\text { psicofísica e genética) }\end{array}$} & Neurobiologia & IBCCF-UFRJ \\
\hline & Fisiologia & ICB-USP \\
\hline & Psic. Experimental & IP-USP \\
\hline & Fisiologia & CCB-UFPA \\
\hline & Psicologia & UFPA \\
\hline & Psicologia & FFCLRP-USP \\
\hline & Psicologia & UFPE \\
\hline & Psicologia & UnB \\
\hline \multirow{4}{*}{ Comportamento animal e neuroetologia } & Psic. Experimental & IP-USP \\
\hline & Zoologia & UFMG \\
\hline & Psicobiologia & UFRN \\
\hline & Psicologia & UFSC \\
\hline \multirow{4}{*}{ Epilepsia } & Fisiologia, Neurologia & UNIFESP \\
\hline & Psicobiologia & UNIFESP \\
\hline & Psicobiologia & FFCLRP-USP \\
\hline & Fisiologia & UFPR \\
\hline \multirow{4}{*}{$\begin{array}{l}\text { Organização funcional do sistema nervoso (morfologia, } \\
\text { células tronco, plasticidade) }\end{array}$} & Neurobiologia & IBCCF-UFRJ \\
\hline & Anatomia & ICB-UFRJ \\
\hline & Fisiologia e Biofísica & ICB-USP \\
\hline & Fisiologia & CCB-UFPA \\
\hline \multirow{3}{*}{ Nutrição } & Fisiologia & UFPE \\
\hline & Fisiologia & CCB-UFPA \\
\hline & Psicobiologia & FFCLRP-USP \\
\hline \multirow{3}{*}{ Sono e Cronobiologia } & Psicobiologia & UNIFESP \\
\hline & Fisiologia Clínica & FM-USP \\
\hline & Fisiologia e Biofísica & ICB-USP \\
\hline \multirow{4}{*}{ Nutrição, desenvolvimento e funções neurais } & Nutrição & UFPE \\
\hline & Neurobiologia & IBCCF-UFRJ \\
\hline & Neurologia & UNIFESP \\
\hline & Psicobiologia & FFCLRP-USP \\
\hline \multirow{3}{*}{ Doença mental (esquizofrenia, doença do pânico) } & Psiquiatria & FM-USP \\
\hline & Farmacologia & UFSC \\
\hline & Psiquiatria & FMRP-USP \\
\hline \multirow{5}{*}{ Neurociência computacional } & \multirow{5}{*}{$\begin{array}{l}\text { Eletricidade } \\
\text { Ciência Computacional }\end{array}$} & EP-USP \\
\hline & & IME-USP \\
\hline & & IF-USP \\
\hline & & FFCL-USP-RP \\
\hline & & IQF-USP-SC \\
\hline \multirow{5}{*}{$\begin{array}{l}\text { Neuroregeneração em acidente vascular cerebral e doença } \\
\text { de Parkinson }\end{array}$} & Biologia celular & ICB-USP \\
\hline & Anatomia & ICB-UFRJ \\
\hline & Neurobiologia & IBCCF - UFRJ \\
\hline & Neurologia & UNIFESP \\
\hline & Bioquímica & UFRGS \\
\hline
\end{tabular}


de Psicologia da Universidade Federal de Pernambuco (UFPE) e, mais recentemente, no Departamento de Fisiologia do Centro de Biologia da UFMG e no grupo de Psicologia da UFPA.

- Organização funcional do sistema nervoso e sua plasticidade, desde o desenvolvimento, apoptose, regeneração até mudanças comportamentais, são tópicos de pesquisa de grupos muito produtivos no Instituto de Biofísica Carlos Chagas Filho da UFRJ, no Instituto de Ciências Biomédicas da USP, no Departamento de Psicobiologia da Faculdade de Filosofia, Ciências e Letras da USP-RP, no Centro de Biologia da UFPA e no Departamento de Psicobiologia da UNIFESP.

- Sono e cronobiologia são também áreas de pesquisa muito ativas no Brasil, com grupos no Departamento de Psicobiologia da UNIFESP, no Instituto de Ciências Biomédicas da USP e no Departamento de Psicobiologia da UFRN.

- Doença mental (síndrome de pânico, esquizofrenia, depressão) e doenças neurodegenerativas são estudadas nos Departamentos de Psiquiatria e de Neurologia da Faculdade de Medicina da USP, que também utilizam modelos animais, nos departamentos de Psiquiatria da UNIFESP, de Fisiologia do Instituto de Ciências Biológicas da Universidade do Estado do Rio de Janeiro (UERJ) e de Neurologia da UNICAMP.

- Engenharia biomédica e redes neurais, estudadas por vários grupos no Instituto de Física, na Escola Politécnica, no Instituto de Matemática e Estatística e na Faculdade de Medicina da USP, na Faculdade de Filosofia, Ciências e Letras da USP-RP, assim como no Instituto de Química e Física da USP em São Carlos (USP-SC).

- Nutrição e funcionamento do cérebro, incluindo eletrofisiologia, morfologia e comportamento em modelos animais de subnutrição são aspectos pesquisados pelos Departamentos de Nutrição e de Fisiologia da UFPE, em interação com outros centros como o Departamento de Neurobiologia do Instituto de Biofísica da UFRJ, o Departamento de Neurologia da Faculdade de Medicina da UNIFESP, o Departamento de Fisiologia do Centro de Ciências Biológicas da UFPA e o Departamento de Psicobiologia da Faculdade de Filosofia, Ciências e Letras da USP-RP.

- Regeneração do sistema nervoso é estudada por meio de procedimentos de estimulação de neurogênese, de neuroproteção e de engenharia tecidual a partir de células pluripotentes. Esses estudos estão sendo conduzidos por grupos pioneiros da USP, da UNIFESP e da UFRJ.

A comunidade de neurocientistas compreende cerca de 2000 pesquisadores, incluindo estudantes em todos os níves, da iniciação científica à pós-graduação e ao pósdoutoramento. Há programas de pós-graduação em Neurociências e Comportamento com este nome ou com o nome de Psicobiologia, na UFRN, na UFSC, na USP, na Faculdade de Filosofia, Ciências e Letras da USP-RP e na UNIFESP. Outros programas como os de ciências morfológicas, bioquímica, biofísica, farmacologia, fisiologia, nutrição e psicologia também incluem orientação em neurociência.
Como consequência, um importante contingente de jovens pesquisadores altamente qualificados nos vários ramos da neurociência tem sido formado na última década. Estima-se que $20 \%$ da produção científica brasileira da área biológica e biomédica seja de neurociência.

É intensa a produção científica da área de pesquisa básica, como se pode verificar em diversos congressos. Na Reunião Anual da Federação de Sociedades de Biologia Experimental (FeSBE), os trabalhos da divisão Neurociências e Comportamento (SBNec) constituem cerca de $30 \%$ do total. O número de trabalhos inscritos no período 2003 a 2007 e no ano de 2009 somou 4476, ou seja, 746, em média (ver Figura 1 e Tabela 3). Mas na reunião da FeSBE existem outras divisões que incluem estudos do sistema nervoso (e.g., toxicologia, neurofarmacologia etc), tornando esse número uma subestimação do total de trabalhos em pesquisa básica em neurociência anualmente apresentados nessa reunião.
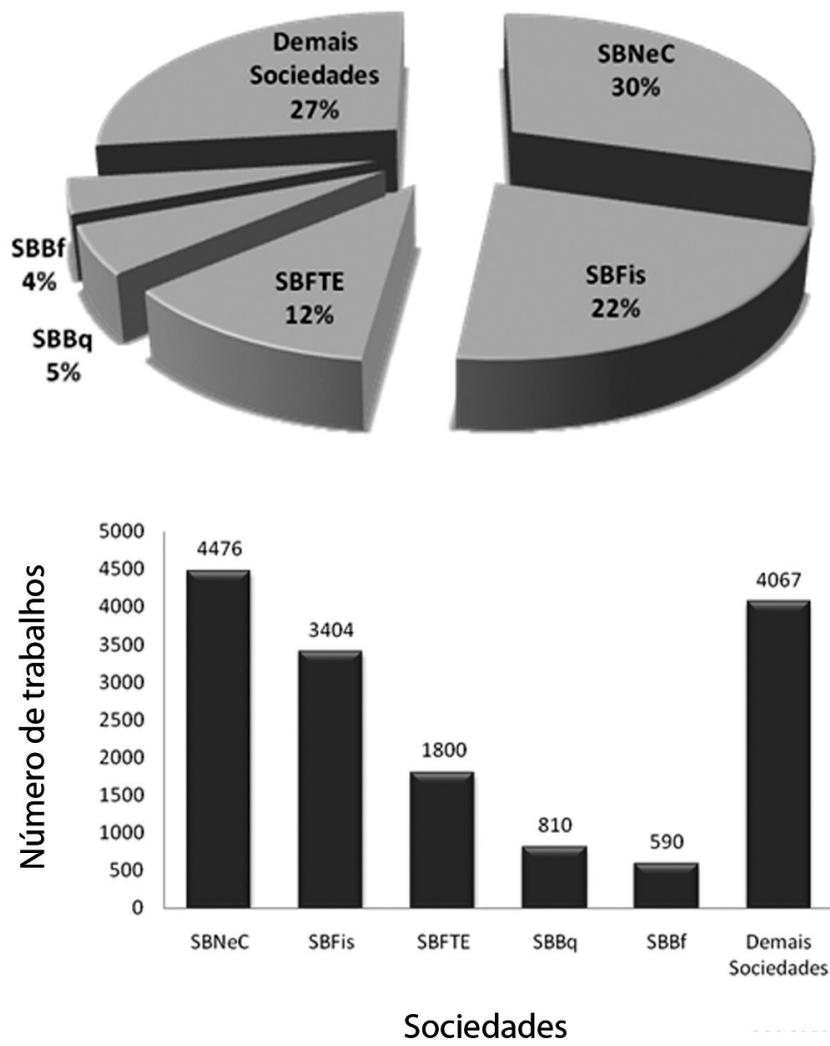

Figura 1. Participação das sociedades afiliadas à Federação de Sociedades de Biologia Experimental (FeSBE) na reunião anual. Dados acumulados dos anos 2003 a 2007 e 2009. Em 2008, a SBNeC organizou seu congresso junto com as sociedades da Argentina, Chile e Uruguai, no Neurolatam (ver texto). SBNeC - Sociedade Brasileira de Neurociências e Comportamento; SBFis - Sociedade Brasileira de Fisiologia; SBFTE - Sociedade Brasileira de Farmacologia e Terapeutica Experimental; SBBq - Sociedade Brasileira de Bioquímica; SBBf - Sociedade Brasileira de Biofísica (dados gentilmente cedidos em 16/09/2010 por Marcus Vinícius Baldo, Presidente da SBNeC)

Outras importantes reuniões científicas também incluem pesquisas em neurociência. Dentre os mais de 1500 trabalhos apresentados na reunião anual da Sociedade Brasileira de Bioquímica e dentre os quase 800 apresentados na reunião da Sociedade Brasileira de Imunologia, uma considerável porção também trata de temas de neurociência. Dentre os trabalhos 
Tabela 3. Proporções das linhas de pesquisa em neurociência apresentadas na Reunião Anual da Federação de Sociedades de Biologia Experimental (FeSBE).

\begin{tabular}{lc}
\hline Linhas de Pesquisa & Proporção \\
\hline Ansiedade, depressão, agressão, defesa & 14,2 \\
Função autonômica & 10,2 \\
Aprendizagem e memória & 8,5 \\
Controle motor & 7,0 \\
Visão e audição & 6,8 \\
Dor & 6,7 \\
Controle neuroendócrino & 5,7 \\
Receptores, transmissores e neuromoduladores & 5,6 \\
Ritmos biológicos & 5,3 \\
Abuso de drogas & 4,5 \\
Epilepsia & 3,6 \\
EEG e potencial evocado & 2,3 \\
Crescimento, diferenciação e morte celular & 1,8 \\
Demais linhas & 18,8 \\
\hline
\end{tabular}

apresentados na Sociedade Brasileira de Psicologia existe uma parcela dedicada à neurociência. A área está também fortemente representada em suas aplicações clínicas, na Sociedade Brasileira de Neuropsicologia. Na Associação Nacional de Pesquisa e Pós-Graduação em Psicologia (ANPEPP) foi criado há 10 anos o grupo de trabalho Psicobiologia, Neurociências e Comportamento, e a partir desse grupo surgiu a revista online Psychology \& Neuroscience, publicada desde 2008. Em 2009, foi criado o Instituto Brasileiro de Neurociências e Comportamento, cuja primeira reunião ocorrerá em outubro de 2010, no Rio de Janeiro.

Segundo o Institute for Scientific Information (http:// www3.isi.edu/home), a neurociência está dentre as mais bem sucedidas áreas de investigação no Brasil, com $20 \%$ dos neurocientistas brasileiros situados dentre os pesquisadores mais produtivos de todas as áreas do conhecimento no Brasil.

\section{Integração Latinoamericana}

Em 2008, realizou-se o I NEUROLATAM - I Congresso de Neurociências Latinoamericano, Caribenho e Ibérico, em Búzios, RJ. Esse congresso reuniu 2600 participantes de 13 países. A expectativa dos organizadores, de cerca de 1000 participantes, foi superada muito antes da data do congresso e as inscrições precisaram ser fechadas com vários meses de antecedência. A idéia do NEUROLATAM surgiu na reunião anual da FeSBE, ocorrida em 2006, na qual o Comitê Regional Latino-Americano (do inglês, Latin American Regional Comittee - LARC) da International Brain Research Organization (IBRO) promoveu, juntamente com a $\mathrm{SBNeC}$, um encontro oficial entre sociedades de neurociências da América Latina, Caribe e Península Ibérica. Os presidentes dessas sociedades e seus representantes (Conselho Ibero-Latino Americano de Presidentes e Representantes de Sociedades de Neurociências e LARC) reconheceram a necessidade premente de promover atividades integrativas e que alavancassem esta área do conhecimento, a começar por um grande congresso que se realizaria em 2008 abarcando todas as sociedades. Esse congresso, por sua vez, deu origem à fundação da Federação de Associações Latinoamericanas de Neurociências (FALAN).

Os congressos da SBNeC ocorreram, a partir de sua fundação em 1977, primeiramente dentro da Sociedade Brasileira para o Progresso da Ciência (SBPC), e desde 1992 passaram a se realizar dentro da FeSBE. Em setembro de 2010, a SBNeC organizou pela primeira vez seu congresso de forma independente, fora da reunião da FeSBE. Esse congresso contou com mais de 1500 inscritos. Embora a ideia de vários congressos simultâneos dentro do guarda chuva da FeSBE tenha a grande vantagem de proporcionar um ambiente multidisciplinar, a realização independente do congresso da $\mathrm{SBNeC}$ é consequência de sua maturidade e volume de associados e atividades.

\section{O Futuro}

As diversas áreas representadas nas neurociências brasileiras surgiram de forma espontânea, de meados do século passado para cá, refletindo oportunidades de treinamento em centros do exterior para os pioneiros da área. Sua diversificação cobre uma razoável extensão do campo das neurociências, embora ainda existam muitas lacunas. Mais do que cobrir todas as lacunas, contudo, devemos pensar no que seria relevante procurar desenvolver em nosso meio. Propomos, a seguir, alguns objetivos que poderão nortear futuras direções de desenvolvimento.

\section{Objetivo 1. Preservação e incentivo às linhas de pesquisa existentes}

Em primeiro lugar, devemos ampliar e fortalecer as linhas de pesquisa já bem estabelecidas e dedicar esforços para a expansão das linhas emergentes que já se instalaram. Em um país em que a massa crítica de pesquisadores é ainda muito pequena, devem ser aproveitados os recursos humanos com que já contamos. Existem também muitos cientistas brasileiros no exterior, destacando-se e realizando pesquisa de fronteira. $\mathrm{O}$ retorno desses cientistas deveria figurar em nossas prioridades.

\section{Objetivo 2. Incorporação da revolução trazida pela genética}

Seja nas linhas existentes, seja em novos programas, a pesquisa em neurociências deverá, em futuro imediato, considerar e incorporar os novos avanços da genética, em consonância com o que está ocorrendo no plano internacional. Esses avanços, os quais tocam em muitas linhas de pesquisa da neurociência, foram obtidos a partir do estudo de novas drogas para doenças neurológicas; das bases genéticas de doenças mentais como esquizofrenia e transtorno maníaco depressivo, incluindo investigacões dos genes desses pacientes que influenciam a resposta a drogas terapêuticas; dos defeitos congênitos; de doenças herdadas e do desenvolvimento como a síndrome do X frágil, síndrome de Rett e o autismo; da genética da adição a drogas e da farmacogenética, que 
avalia a influência dos genes do indivíduo na sua resposta a medicamentos (e.g., antidepressivos).

\section{Objetivo 3. Incentivo à pesquisa clínica}

Para a tradução dos novos avanços genéticos ou de novos medicamentos e procedimentos em geral, em métodos terapêuticos e de diagnóstico, há necessidade de se incentivar a pesquisa translacional realizada por meio da pesquisa clínica com seres humanos. Isso vale não apenas para a neurociência, mas para toda a pesquisa biomédica. $\mathrm{Na}$ pesquisa biomédica, além de protocolos de avaliação dos efeitos de medicamentos, os estudos sobre envelhecimento são muito relevantes, dado o contínuo aumento na expectativa de vida. Os EUA estão investindo na pesquisa sobre retardamento do aparecimento e da evolução da doença de Alzheimer ou, até mesmo, da prevenção dessa patologia, com grandes estudos clínicos usando vitamina $\mathrm{E}$, a droga Aricept, drogas antiinflamatórias e estrógeno. Outros estudos estão focalizando a busca de fatores ambientais responsáveis pela doença de Parkinson, como pesticidas, solventes e metais e avaliando drogas que lentificam a progressão da doença. São ainda muito importantes em nosso meio os estudos de neuropatologias regionais. Numa situação em que a pobreza da população está acompanhada pela desnutrição e por más condições sanitárias, patologias erradicadas ou inexistentes em países avançados são prevalentes no Brasil. Aspectos neurológicos de várias doenças degenerativas tropicais, como a malária e outras, deveriam ser estudados por nossos cientistas.

O NIMH (2001), preocupado com impactos da doença mental na inclusão social desenvolveu uma rede de centros de excelência dedicados à pesquisa básica e clínica em autismo para prevenir, diagnosticar e tratar esta e outras doenças mentais em crianças. No Brasil, está sendo desenvolvido pela Academia Brasileira de Ciências um projeto do Centro de Apoio a Moradias Assistidas e ao Trabalho (CAMT), que se dedica a pesquisar causas e tratamentos das patologias, bem como a implantação e o gerenciamento de moradias assistidas para os pacientes.

\section{Objetivo 4. A incorporação de novas tecnologias}

A área de pesquisas de neuroimagem e, em especial, a ressonância magnética funcional, precisa se desenvolver nos próximos anos, em nosso meio, sob pena de ficarmos em grande defasagem com os países desenvolvidos. Essas novas tecnologias não invasivas estão rapidamente esclarecendo não só muitos aspectos do funcionamento normal do sistema nervoso, como são potencialmente úteis na compreensão de patologias. Por exemplo, a ressonância magnética funcional foi aplicada em estudos do National Institute on Drug Abuse (NIDA, 2010), pertencente ao NIH, usando, para examinar o desenvolvimento cerebral de bebês expostos durante a gestação, a metanfetamina e outras drogas que afetam a ação normal de neurotransmissores. Uma revisão sobre neurociência e alcoolismo, elaborada pelo National Institute of Alcohol Abuse and Alcoholism (NIAAA, 2008a, 2008b) mostra os efeitos do álcool no cérebro e as contribuiçôes da neurociência na elucidação dos circuitos envolvidos nas adaptações biológicas ao álcool.

$\mathrm{Na}$ área de neuroimagem temos ainda pouca atividade, apesar de já existirem excelentes recursos humanos, que estão constituindo núcleos formadores. Há necessidade de investimento planejado nessa área e, em geral, nos métodos diagnósticos não invasivos de alta tecnologia que têm surgido nas neurociências. Para tanto, devem ser constituídas equipes multidisciplinares, com forte ênfase em profissionais de informática, além de clínicos e pesquisadores básicos das áreas de neurofisiologia e psicologia experimental.

Em conclusão, o presente documento procura trazer um retrato da área de neurociências no Brasil no momento, mostrando sua potencialidade e sugerindo caminhos para o futuro. Esse futuro depende naturalmente do apoio constante e integral das agências de fomento, seja em programas especiais, seja na manutenção e ampliação dos programas de fomento gerais que têm existido.

\section{Referências}

Library of Congress - LOC (2010). Project on the decade of the brain. Retirado em 16/09/2010, de http://www.loc.gov/loc/brain.

National Institute of Alcohol Abuse and Alcoholism - NIAAA (2008a). Neuroscience: Pathways to alcohol dependence - Part I: Overview of the Neurobiology of Dependence. Retirado em 14/09/2010, de http://pubs.niaaa.nih.gov/publications/ arh313/ toc31-3. htm.

National Institute of Alcohol Abuse and Alcoholism - NIAAA (2008b). Neuroscience: Pathways to alcohol dependence-Part II: Neuroadaptation, risk, and recovery. Retirado em 14/09/2010, de http://pubs.niaaa.nih.gov/publications/ arh313/toc31-3.htm.

National Institute of Health - NIH (2002). Disease-specific estimates of direct and indirect costs of illness and NIH Support - NINDS. Retirado em 19/09/2010, de http://www.ninds.nih.gov/ news_and_events/congressional_testimony/2002_appropriation justification.htm.

National Institute of Mental Health - NIMH (2001). Five-year strategic plan for reducing health disparities. Retirado em 16/09/2010, de http://www.nimh.nih.gov/about/strategic-planning-reports/nimhfive-year-strategic-plan-for-reducing-health-disparities.pdf.

National Institute of Mental Health - NIMH (2002). Pathways to health: Charting the science of brain, mind, and behavior. Retirado em 16/09/2010, de http://www.nimh.nih.gov/about/strategicplanning-reports/index.shtml

National Institute on Drug Abuse - NIDA (2010). Drugs that may have adverse prenatal effects. fMRI and the effects of prenatal methamphetamine exposure on verbal memory. Retirado em 16/09/2010, de http://www.nida.nih.gov/consequences/prenatal/.

Silveira, L. C. L. (2004). Neurociências no Brasil - uma revolução tecnológica ao nosso alcance. Neurociências, 1, 42-47.

Timo-Iaria, C. (s.d.). História da neurofisiologia no Brasil. Retirado em 15/9/2010, de http://www.sbnec.org.br.

Ventura, D. F. (1997). Report on experimental biology in Brazil. Em L. Bevilacqua (Org.), Science in Brazil - An overview (pp. 1631). Rio de Janeiro: Academia Brasileira de Ciências.

World Health Organization - WHO (2002). Global burden of disease - GBD. Retirado em 16/09/2010, de http://www.who.int/ healthinfo/global_burden_disease/en/index.html. 